Article

\title{
Quartz Enhanced Photoacoustic Spectroscopy Based on a Custom Quartz Tuning Fork
}

\author{
Maxime Duquesnoy ${ }^{1,2, *} \mathbb{0}$, Guillaume Aoust ${ }^{1}$, Jean-Michel Melkonian ${ }^{2}$, Raphaël Lévy ${ }^{2}$, \\ Myriam Raybaut ${ }^{2, *(1)}$ and Antoine Godard ${ }^{2}(\mathbb{D})$ \\ 1 Mirsense, Nanno-INNOV, building 863, 8 Avenue de la Vauve, 91120 Palaiseau, France; \\ guillaume.aoust@mirsense.com \\ 2 DPHY, ONERA, Université Paris Saclay, F-91123 Palaiseau, France; \\ jean-michel.melkonian@onera.fr (J.-M.M.); Raphael.Levy@onera.fr (R.L.); antoine.godard@onera.fr (A.G.) \\ * Correspondence: maxime.duquesnoy@mirsense.com (M.D.); myriam.raybaut@onera.fr (M.R.); \\ Tel.: +33-180-38-61-77 (M.D.); +33-180-38-64-38 (M.R.)
}

Received: 14 February 2019; Accepted: 14 March 2019; Published: 19 March 2019

check for updates

\begin{abstract}
We have designed and fabricated a custom quartz tuning fork (QTF) with a reduced fundamental frequency; a larger gap between the prongs; and the best quality factor in air at atmospheric conditions ever reported, to our knowledge. Acoustic microresonators have been added to the QTF in order to enhance the sensor sensitivity. We demonstrate a normalized noise equivalent absorption $(N N E A)$ of $3.7 \times 10^{-9} \mathrm{~W} \cdot \mathrm{cm}^{-1} \cdot \mathrm{Hz}^{-1 / 2}$ for $\mathrm{CO}_{2}$ detection at atmospheric pressure. The influence of the inner diameter and length of the microresonators has been studied, as well as the penetration depth between the QTF's prongs. We investigated the acoustic isolation of our system and measured the Allan deviation of the sensor.
\end{abstract}

Keywords: QEPAS; photoacoustic spectroscopy; quartz tuning fork

\section{Introduction}

Detection of hazardous chemicals and greenhouse gases is one among the many challenges of our century. One of the difficulties lies in the fact that the gases that we need to detect are at trace level, from a few percent to a few ppbv (part per billion in volume), with large variations of the concentration in time. The ability to detect several gases with a single detector is a highly desirable feature, but is hard to achieve. In addition, to be deployed in the field, it is convenient to have a detection technique allowing one to keep a good sensitivity while reducing the overall volume and with a low power consumption. A technique meeting all these requirements is photoacoustic spectroscopy-it presents a wide dynamic range and a very good linearity, and enables multi-species detection with a single and compact device. In this technique, a pressure wave is generated by relaxation of the gas after it has been excited by absorbed modulated light through molecular transitions [1].

The sensitivity of a photoacoustic detector can be qualified by a figure of merit, called normalized noise equivalent absorption (NNEA). It represents the minimum detectable absorption, independent from the laser power, the electrical bandwidth, and the absorption coefficient of the gas. It is defined as follows:

$$
N N E A=\frac{P \times \alpha}{S N R \times \sqrt{\Delta f}}
$$

where $P$ is the average optical power in $\mathrm{W} ; \alpha$ is the target gas absorption in $\mathrm{cm}^{-1} ; \Delta f$ is the detection bandwidth in $\mathrm{Hz}$; and SNR is the signal to noise ratio, taken as the ratio of the signal root mean square (RMS) value with the RMS noise within the same bandwidth. 
A new technique called quartz enhanced photoacoustic spectroscopy (QEPAS) was introduced in 2002 [2], providing a good tradeoff between compactness and sensitivity of the acoustic detector, as well as good acoustic isolation from the environment. This technique involves the generation of a pressure wave through gas excitation with an amplitude- or wavelength -modulated laser and its detection with a quartz tuning fork (QTF), usually complemented by longitudinal acoustic resonators.

\subsection{Commercial Quartz Tuning Forks}

The first available QTFs came from the watch industry. They are generally used under vacuum for time reference applications, but also in air for atomic force spectroscopy [3], for example. However, they present two major drawbacks for photoacoustic spectroscopy-a small gap between their prongs $(\sim 200-300 \mu \mathrm{m})$ and a high resonance frequency $\left(32,768 \mathrm{~Hz}\right.$ corresponding to $\left.2^{15} \mathrm{~Hz}\right)$.

Indeed, the small gap between their prongs makes it difficult for a laser beam to pass through. It is even more critical with mid-infrared (MIR) lasers presenting larger, diffraction-limited, beam waists, like quantum cascade lasers (QCL) for instance. This spectral band, ranging from 3 to $50 \mu \mathrm{m}$, is nevertheless of high interest for gas sensing, as it is called the "molecular fingerprint region" for many targeted gases. To circumvent this limitation, one can resort to hollow core fibers to facilitate QCL beam alignment between the QTF's prongs, leading to a more complex optical setup [4].

A high resonance frequency will make measurements less sensitive, if not impossible, for gases presenting slow vibrational-translational times, such as $\mathrm{CO}_{2}$ in the near infrared or MIR. For example, at $2 \mu \mathrm{m}, \mathrm{CO}_{2}$ exhibits a relaxation time of a few microseconds [5], leading to a signal reduction of $60 \%$ for a $32,768 \mathrm{~Hz}$ tuning fork.

In addition to these drawbacks, standard QTFs present low-quality factors at atmospheric pressure $(Q \sim 10,000)$ compared with their quality factor under vacuum $(Q \sim 100,000)$. As the SNR scales as the root mean square of the QTF's quality factor, it is possible to enhance the sensor sensitivity by improving the detector's quality factor.

Nonetheless, the use of classical watch QTFs allowed to reach a NNEA of $3.3 \times 10^{-9}$ W.cm ${ }^{-1} \cdot \mathrm{Hz}^{-1 / 2}$ for $\mathrm{C}_{2} \mathrm{H}_{2}$ at atmospheric pressure, with the use of adapted longitudinal acoustic resonators [6]. Similar values have been reported in almost all of the recent papers on the topic [6,7], even if the best value of $3.75 \times 10^{-11} \mathrm{~W} . \mathrm{cm}^{-1} \cdot \mathrm{Hz}^{-1 / 2}$ was reported in 2016 [8]. In comparison, classical PAS sensitivity with acoustical resonators and microphones demonstrates similar sensitivities with a best reported NNEA of $2.4 \times 10^{-10}$ W.cm ${ }^{-1} \cdot \mathrm{Hz}^{-1 / 2}$ [9].

\subsection{Custom Quartz Tuning Forks}

In order to mitigate these drawbacks, a few custom QTFs have been reported in the literature [10-12], presenting T-shape prongs, a larger gap between the prongs from 0.4 to $1 \mathrm{~mm}$, reduced resonance frequency down to $2.8 \mathrm{kHz}$, and enhanced quality factor in air at atmospheric pressure of 15,000 at best. These resonators were demonstrated to work at the first overtone frequency and making possible dual gas sensing by exciting both fundamental and overtone flexural modes [13]. Another choice is to reduce the gap between the prongs to benefit more from the acoustic pressure actuating the QTF [14], despite the induced elevation of the background noise due to higher residual illumination of the QTF's prongs. This approach could be considered in the MIR thanks to the use of background noise suppression techniques such as the electrical modulation cancellation method [15] or phase quadrature measurements, which we recently introduced [16]. It is also possible to use different configurations such as off-beam QEPAS to avoid illumination of the QTF's prongs by the laser $[17,18]$.

\subsection{Our Custom Quartz Tuning Fork}

In previous works $[19,20]$, we introduced our own custom QTF (Figure 1) with adapted thin film gold electrodes, presenting a gap between the prongs of $2 \mathrm{~mm}$ and a frequency of 21,500 Hz. The QTF is $2 \mathrm{~mm}$ thick, and its prongs are $13.6 \mathrm{~mm}$ high and $8 \mathrm{~mm}$ large. 


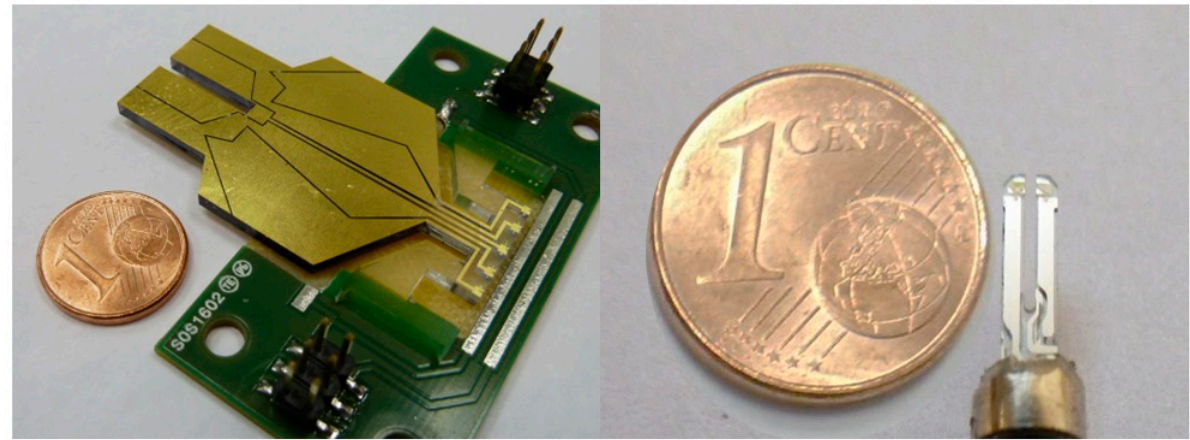

a)

b)

Figure 1. (a) Picture of the custom quartz tuning fork used for our experiment. The quartz tuning fork (QTF) presents a $2 \mathrm{~mm}$ gap between the prongs. (b) Picture of a commercial fork.

This QTF, called C2, was optimized by the use of an analytical model [20] and through finite element modeling (FEM) using OOFELIE::Multiphysics@ [21]. The QTF frequency was a result of the optimization of the quality factor and is still high for photoacoustics. However, we already presented another QTF presenting a lower frequency of $5 \mathrm{kHz}$ [22], allowing us to measure gases with slow relaxation times. C2 presents a quality factor of 450,000 under vacuum and 7600 in air at atmospheric pressure [19], which is very close to the predicted value of 7320, as shown in Table 1. As explained hereafter in detail, this tuning fork was designed to be placed within a specific acoustic cavity, leading to this moderate $Q$ factor for the bare QTF.

Table 1. Theoretical performances of our custom quartz tuning fork (QTF) [19].

\begin{tabular}{ccc}
\hline & Bare QTF & QTF + Acoustic Recovery \\
\hline Frequency & $21.23 \mathrm{kHz}$ & $21.23 \mathrm{kHz}$ \\
$Q_{\text {support }}$ & $1 \times 10^{6}$ & $1 \times 10^{6}$ \\
$Q_{\text {thermo }}$ & $5.48 \times 10^{7}$ & $5.48 \times 10^{7}$ \\
$Q_{\text {squeeze }}$ & $3.82 \times 10^{7}$ & $3.82 \times 10^{7}$ \\
$Q_{\text {viscous }}$ (lateral) & $2.91 \times 10^{5}$ & $2.91 \times 10^{5}$ \\
$Q_{\text {viscous }}$ (frontal) & $2.96 \times 10^{5}$ & $2.96 \times 10^{5}$ \\
$Q_{\text {acoustic }}$ & $7.76 \times 10^{3}$ & - \\
$\mathbf{Q}$ total & $\mathbf{7 . 3 2} \times \mathbf{1 0}^{\mathbf{3}}$ & $\mathbf{1 . 2 7} \times \mathbf{1 0}^{\mathbf{5}}$ \\
\hline
\end{tabular}

The total quality factor predicted by our analytical model accounts for anchor losses $Q_{\text {support }}$, thermoelastic damping of the material $Q_{\text {thermo }}$, squeezing $Q_{\text {squeeze }}$ due to acoustic waves confinement between structures, viscous losses $Q_{\text {viscous }}$ (both lateral and frontal) due to dissipation into the fluid, and acoustic radiation $Q_{\text {acoustic }}$. In contrast with classical QTFs, C2 introduces an important energy loss through acoustic radiation. This loss can be expressed as follows through its associated quality factor [20]:

$$
Q_{\text {acoustic }}=\frac{64}{3} \frac{\rho_{q}}{\rho_{f}} \frac{1}{k_{v}^{4} e l(e+g)^{2}},
$$

where $\rho_{q}$ is the quartz volumic density, $\rho_{f}$ is the fluid volumic density, $k_{v}$ is the acoustic wave vector, $e$ is the QTF branch width, $g$ is the gap between its prongs, and $l$ is the QTF thickness.

To recover this radiated energy, an enclosing cavity was added to our QTF to confine the acoustic waves emitted by the QTF itself [19], providing an unmatched quality factor of 45,400 in air, around $21,500 \mathrm{~Hz}$, along with a better acoustic isolation from the environment. This value is lower than the theoretical $Q$ factor calculated in Table 1 because of remaining acoustic losses that can be estimated to be $Q_{\text {acoustic }}=75,000$, coming from the complexity to recover the acoustic waves with a perfect phase-match. To our best knowledge, it is the first time such a cavity has been used to recover the acoustic waves emitted by the QTF, in photoacoustic spectroscopy. 
FEM simulations of our system demonstrated that the radiating acoustic pressure field was remarkably cylindrical and in the QTF's plane, as shown in Figure 2. The cavity was thus designed as a cylinder whose radius was adapted through FEM in order to generate acoustic stationary waves in phase with the QTF's emitted acoustic waves. The distance between the prongs and the cavity wall is approximately equal to half the acoustic wavelength $\lambda$.

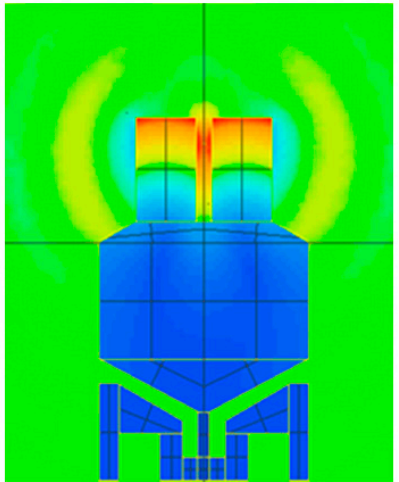

a)

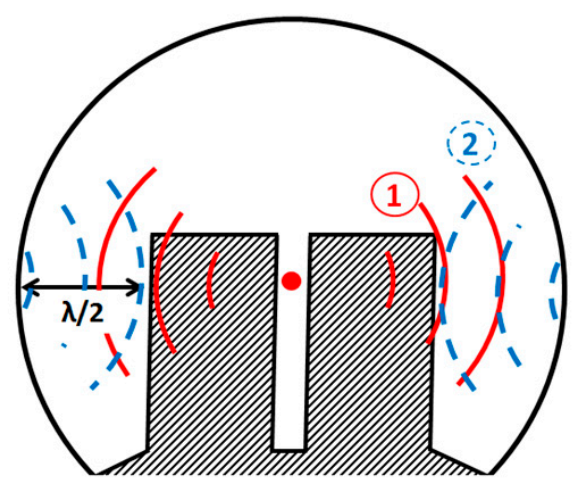

b)

Figure 2. (a) Acoustic radiation of our QTF simulated by finite element modeling (FEM). The QTF was excited by applying a pressure force on the QTF prongs. (b) Principle scheme of acoustic recovery-firstly, emission of acoustic waves by the QTF; and secondly, reflection thanks to the acoustic recovery device.

Finally, acoustic microresonators have been added to our system (Figure 3). This configuration called on-beam [6] allows enhancing the sensor sensitivity by increasing the acoustic pressure near the QTF. Usually two longitudinal acoustic resonators are added to the QTF excited in its fundamental mode providing an increase of 15 to $40[6,23,24]$ on the sensor sensitivity. Unfortunately, this comes along a great diminution of the overall quality factor, which is difficult to predict analytically [25].

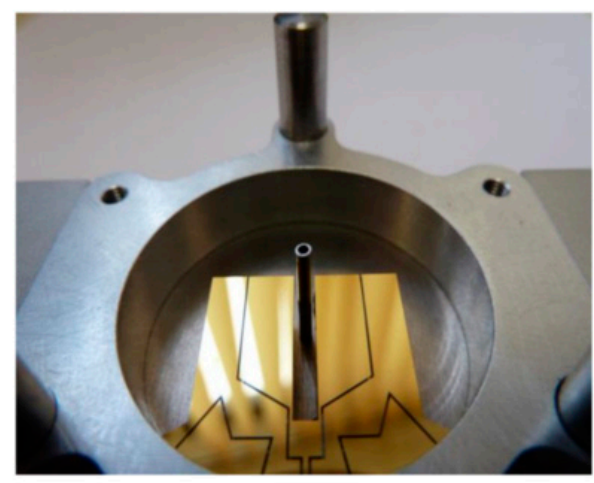

a)

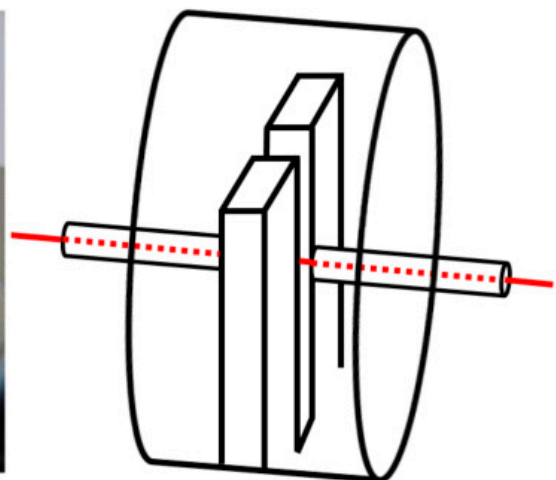

b)

Figure 3. (a) Picture of the QTF within its acoustic cavity: the microresonators for acoustic amplification and the surrounding cylinder for acoustic recovery. (b) Schematic of our QTF used with its acoustic recovery cavity and acoustic microresonators, with a laser beam passing through (in red).

Concerning the electronics, the used circuit is an analog circuit including one transimpedance amplifier, followed by a voltage amplification stage made of two band-pass filters. Measurements of the sensor's noise spectrum and results from a simulation software confirmed that the noise at resonance is given by the Brownian movement of particles. In other words, the sensor noise is limited by its fundamental limit.

In the following paragraphs, we experimentally study the influence of the addition of the previously introduced acoustic devices. 


\section{Influence of Added Acoustic Devices}

\subsection{Environment Isolation by an Acoustic Cavity}

As presented previously, we developed a cavity allowing acoustic recuperation of the acoustic waves emitted by our custom quartz tuning fork (Figure 3). One cavity was designed by FEM-it is a stainless steel cylinder with a $16 \mathrm{~mm}$ radius and a $16 \mathrm{~mm}$ height.

In order to study the isolation of our system to ambient acoustic noise, we placed it in an isolated chamber together with a loud speaker generating a pure sine wave. Without moving the loud speaker, we changed the configuration of our system and measured its response when varying the frequency of the generated sound. Despite the chamber not being anechoic, we expect this experiment to provide representative data on our system, as we do not expect the chamber to have a huge influence on our detector.

In Figure 4, we trace the frequency responses of each configuration after normalizing them by the response of the configuration "QTF alone".

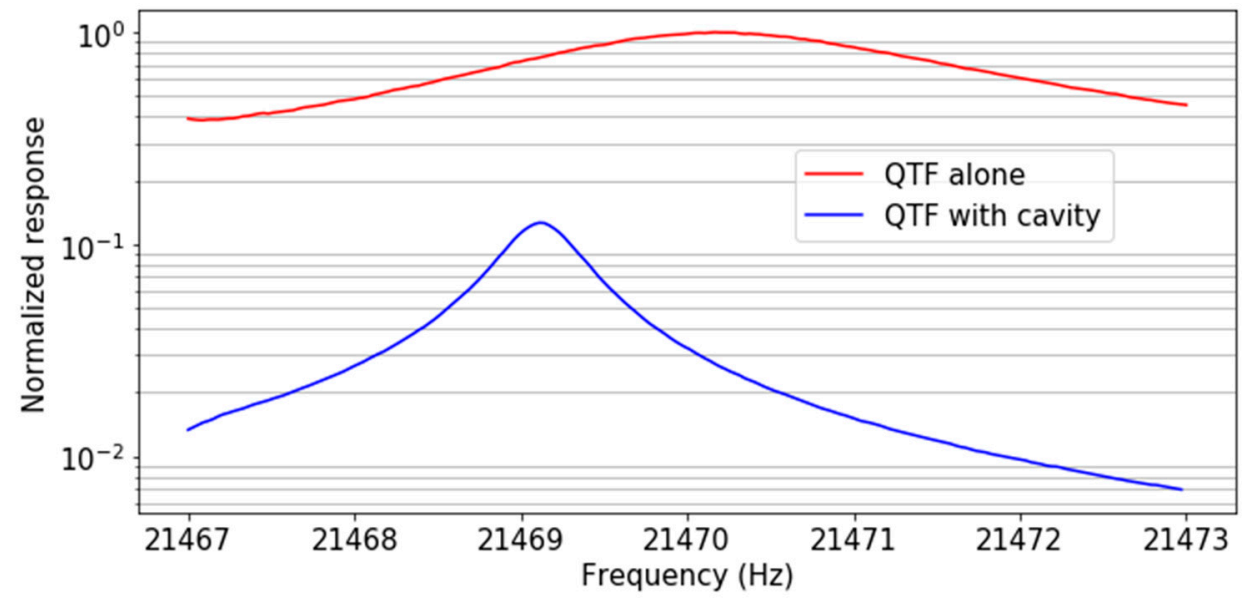

Figure 4. Normalized sensitivity to the external sound, for the bare C2 QTF (red) and for C2 with its acoustic recovery cavity without microresonators (blue).

We also measured the $Q$ factor and resonance frequency with an impedance-meter (4294A, Agilent Technologies, Santa Clara, CA, USA), and obtained the results presented in Table 2.

Table 2. Acoustic isolation granted by our cavity compared with the case in which no cavity is employed.

\begin{tabular}{ccc}
\hline & C2 Alone & C2 with Cavity \\
\hline Frequency $(\mathrm{Hz})$ & $21,470.22$ & $21,469.12$ \\
$Q$ factor & 7600 & 45,441 \\
Normalized sensitivity to external sound & 1 & $1 / 8$ \\
\hline
\end{tabular}

The cavity is promising for the improvement of the system's acoustic isolation. Indeed, a reduction of about eight times of the acoustic signal at the QTF's resonance frequency is observed, along with a quality factor six times better compared with the case in which no cavity is employed. This isolation can be partly explained by the enhancement of the system's overall quality factor, and may also be explained by the acoustic shielding provided by the walls of the acoustic cavity.

\subsection{Design Optimization of the Microresonators}

After having characterized the effect of the cavity on the QTF, we study the influence of the geometry of the microresonators on the quality factor when using such a cavity. These longitudinal 
acoustic resonators are placed on both sides of the QTF's prongs. Their introduction modifies the global quality factor in a non-trivial way [25].

In theory, the optimum tube length would be equal to $\lambda / 2$ if the tubes were decoupled from each other and from the QTF and equal to $\lambda / 4$ if the tubes were totally coupled. In practice, the optimum tube length is somewhere between these two values [6], and has to be determined from FEM simulations or, better, experimentally. In addition, these microresonators should always be chosen with the smallest possible diameter because the acoustic pressure scales inversely with the diameter. On the other hand, the diameter must be large enough for the laser beam to pass through.

Figure 5 shows the system quality factor over inner diameter, length, and penetration depth of the microresonators.

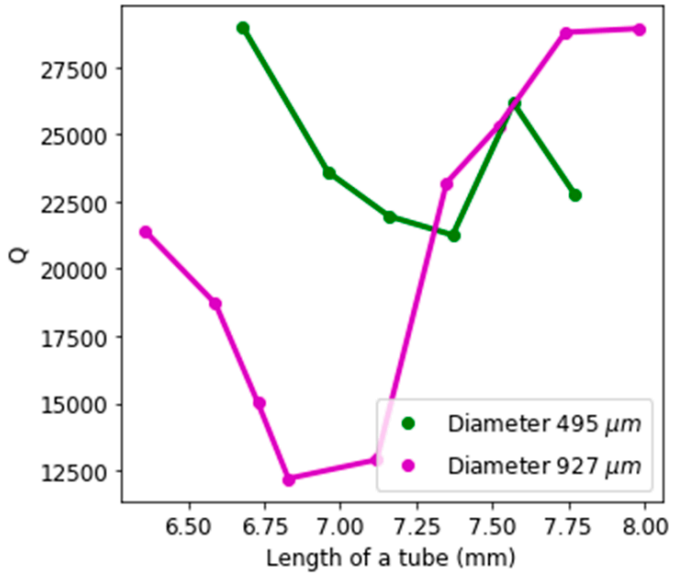

a)

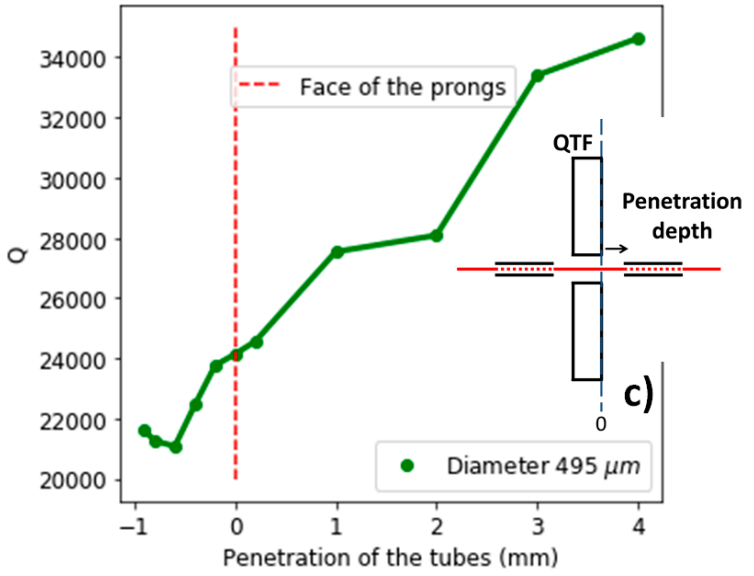

b)

Figure 5. (a) Quality factor of the system (QTF + microresonators) as a function of the length of the tubes, for two different diameters of the tubes and a penetration depth of the tubes of $-0.8 \mathrm{~mm}$ with respect to the face of the prongs. (b) Same as (a) for tubes of same length and diameter, depending on the depth of penetration of the tubes within the gap between the QTF's prongs. The quality factor is measured with an impedance-meter. (c) Schematic of the penetration depth.

When varying the length of the microresonators, a quality factor minimum is identified, corresponding to a maximum acoustic coupling with the QTF. A better coupling comes along with a better acoustic pressure amplification, and thus a better signal-to-noise ratio [6]. In Figure 5b, we see that the $Q$ factor decreases when reducing the distance to the prongs, except between -0.8 to $-0.5 \mathrm{~mm}$, where it increases. The reason for this is a reduced coupling between the resonators when the gap between the needles becomes smaller.

We can conclude that the optimal microresonators should be $7.3 \mathrm{~mm}$ long for an internal diameter of $495 \mu \mathrm{m}$ and a penetration depth of $-0.8 \mathrm{~mm}$. Adding the microresonators to the QTF and acoustic recovery cavity reduces the quality factor to half its initial value. The resulting $Q$ factor of 21,000 is ten times the quality factor obtained for watch QTFs with adapted microresonators [6].

Despite the drop on the quality factor, the addition of such resonators allowed a sensitivity enhancement of about 10, as we will detail hereafter.

\section{Gas Detection}

\subsection{Targeted Absorption Line}

To measure the sensitivity of our system, we use a certified $\mathrm{CO}_{2}$ mixture of $2.7 \%$ in nitrogen. The laser used for the detection is a standard telecom laser diode emitting around $1.5 \mu \mathrm{m}$. We target the $6490.05 \mathrm{~cm}^{-1}$ absorption line of $\mathrm{CO}_{2}$ with an absorbance of $5 \times 10^{-6} \mathrm{~cm}^{-1}$ for a $2.7 \%$ concentration and a full width at half maximum (FWHM) of $0.16 \mathrm{~cm}^{-1}$. The ambient humidity is monitored and 
corresponds to typical atmospheric humidity ( 10,000 ppm), even though water vapor interferes very weakly with our measurement (Figure 6).

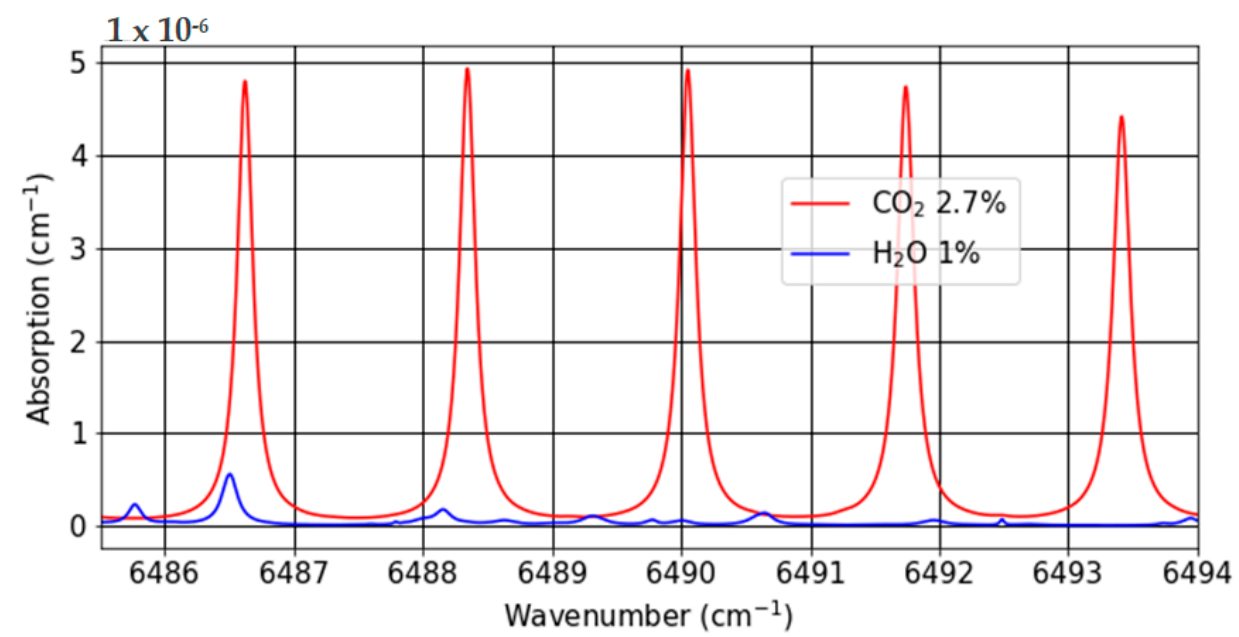

Figure 6. Calculated absorption spectra of $\mathrm{CO}_{2}$ (red) and $\mathrm{H}_{2} \mathrm{O}$ (blue) at atmospheric conditions $(P=$ $101,325 \mathrm{~Pa}, \mathrm{~T}=300 \mathrm{~K})$ using the HITRAN database.

\subsection{Setup}

We use a wavelength modulation scheme with second harmonic detection. The setup is presented in Figure 7. The laser diode temperature and current are controlled (ITC 510, Thorlabs, Newton, MA, USA) and the laser wavelength is modulated with a $f_{0} / 2$ sinusoid wave (33000B, Agilent Technologies, Santa Clara, CA, USA), where $f_{0}$ is the QTF's fundamental vibration frequency. The wavelength is measured continuously with a wavemeter (WS6 785, HighFinesse, Tübingen, Germany) to keep a good match between the absorption line and the laser optical frequency. The QTF's signal is first amplified by means of a low noise transimpedance amplifier, and sent to a lock-in amplifier (SR530, Stanford Research Systems, Sunnyvale, CA, USA) with a 1/16 Hz bandwidth, measuring the second harmonic signal.

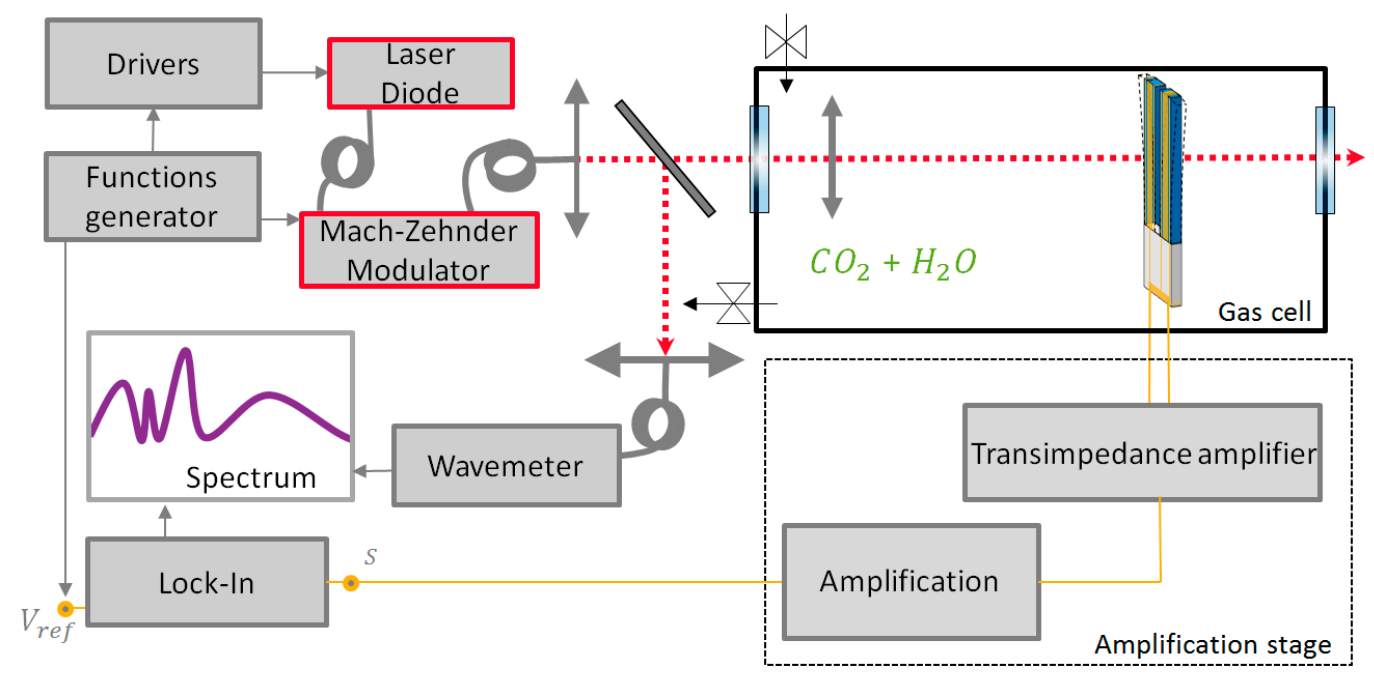

Figure 7. Detection scheme for quartz enhanced photoacoustic spectroscopy (QEPAS) $\mathrm{CO}_{2}$ detection.

The cell contains two wedged windows with an antireflection coating, a lens of focal length $200 \mathrm{~mm}$, the tuning fork, and its transimpedance amplifier. The cell is filled with the gas mixture before each measurement. The custom QTF C2 is surrounded by a $16 \mathrm{~mm}$ radius stainless steel cylinder acting as an acoustic cavity allowing in-phase recovery of the emitted acoustic waves, as explained 
in Section 1.3. Two longitudinal acoustic resonators, which are cut from stainless steel hypodermic needles, are placed along the laser beam, inside the cavity. For these needles, various parameters have been tested: internal diameter, length, position with respect to the QTF stem, and penetration depth within the gap between the prongs, as explained in Section 2.2. We also designed a monolithic cavity including the needles in bulk stainless steel to ease the optical alignment.

\section{3. $\mathrm{CO}_{2}$ Detection}

After having optimized the design of the acoustic microresonators, we performed the detection of $\mathrm{CO}_{2}$ at $2.7 \%$ in nitrogen. The average modulated laser power was measured in front of the QTF, to be $P=17 \mathrm{~mW}$.

By measuring the signal amplitude and the RMS noise within the detection bandwidth, we find a NNEA of $3.3 \times 10^{-8} \mathrm{~W} . \mathrm{cm}^{-1} \cdot \mathrm{Hz}^{-1 / 2}$ for our QTF alone and we reach a sensitivity of $3.7 \times 10^{-9} \mathrm{~W} \cdot \mathrm{cm}^{-1} \cdot \mathrm{Hz}^{-1 / 2}$ for the QTF with the acoustic cavity and the best microresonators cut from needles, providing an enhancement of a factor 10. This enhancement is slightly lower than reported values usually ranging from 15 to $40[6,23,24]$ for on-beam QEPAS using dual tube resonators exciting the QTF's fundamental mode. This might come from the enhanced acoustic coupling between the QTF and the microresonators, as the QTF emits acoustic waves disturbing the microresonators' acoustic resonance.

We also designed the acoustic cavity in a bulk piece of stainless steel with integrated tubes of different sizes, allowing good alignment repeatability. With machined tubes of diameter $0.7 \mathrm{~mm}$ and length $7.3 \mathrm{~mm}$, we obtained a NNEA of $7.6 \times 10^{-9} \mathrm{~W} . \mathrm{cm}^{-1} \cdot \mathrm{Hz}^{-1 / 2}$. The monolithic acoustic cavity could not be machined with microresonators of diameter less than $0.7 \mathrm{~mm}$, because it becomes difficult for suppliers to guarantee the tolerances and a good inner surface polishing. Nonetheless, it would be interesting to carry on in this direction to verify that we can obtain the same results than those obtained with the hypodermic needles.

Finally, an Allan deviation measurement was performed (Figure 8) to analyze the sensor's stability. The C2 QTF was used with both acoustic devices (acoustic recovery and optimal microresonators) to measure the photoacoustic signal in $2.7 \%$ of $\mathrm{CO}_{2}$ at normal conditions of pressure and temperature.

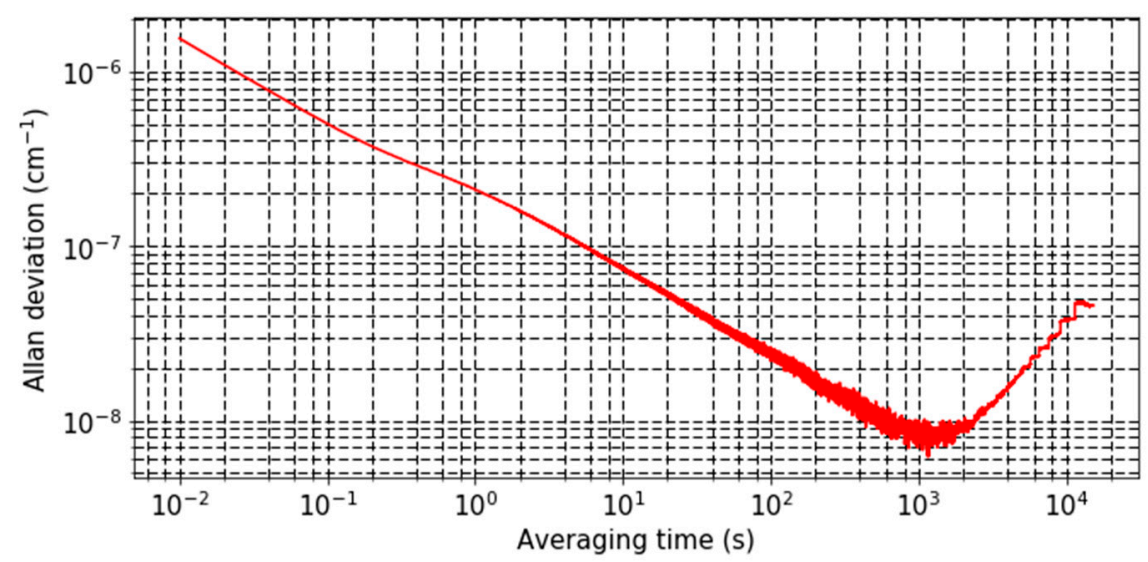

Figure 8. Allan deviation of the measured absorption of $2.7 \%$ of $\mathrm{CO}_{2}$ at $6490.05 \mathrm{~cm}^{-1}$.

We can divide the analysis of the curve in three parts. From $10 \mathrm{~ms}$ to $1 \mathrm{~s}$, we see an averaging surely owing to acoustic phenomena within the acoustic devices, from $1 \mathrm{~s}$ to $1150 \mathrm{~s}$ the averaging of the thermal noise of the QTF and beyond, we see that the sensor is drifting due to temperature induced frequency shift. Compared with other QEPAS measurements [26,27], we see that the drift occurs later-it could be explained by the fact that our QTF is much larger than any other QTF, making it less sensitive to drifts. A minimum deviation of $9 \times 10^{-9} \mathrm{~cm}^{-1}$ is obtained for a 19 min averaging time, corresponding to $44 \mathrm{ppmv}$ of $\mathrm{CO}_{2}$ detected at $1.54 \mu \mathrm{m}$. This concentration value could easily be 
enhanced targeting a stronger absorption line of $\mathrm{CO}_{2}$ at MIR wavelengths (QCLs) and by raising the laser power.

\section{Conclusions}

A quartz-enhanced photoacoustic gas detector with a custom quartz tuning fork was demonstrated. Its lower detection frequency makes it more suitable to address gases with slower relaxation times. Moreover, owing to the wider gap between the QTF's prongs $(2 \mathrm{~mm})$ than for standard QTFs, a better sensitivity and less experimental difficulties are expected with the use of larger laser beams in the mid-infrared (typically from QCLs).

To improve the performance, the custom QTF is enclosed in a cylindrical acoustic cavity with longitudinal acoustic microresonators. The acoustic cavity enables the recovery of the energy radiated though generated acoustic waves, while the acoustic microresonators provide an additional enhancement of the sensitivity through an increase of the pressure between the QTF's prongs. We designed and optimized the acoustic cavity and the acoustic resonators, showing the influence of different parameters of these objects. The best geometry for the tubes was found, with $7.3 \mathrm{~mm}$ length, $0.5 \mathrm{~mm}$ diameter, and penetration depth of $-0.8 \mathrm{~mm}$ inside the QTF's prongs when using a laser diode at telecom wavelength. We also designed bulk cavities with larger needles diameters and optimal lengths to accommodate for larger laser beams as those emitted by MIR lasers.

We showed that the cavity used for acoustic recovery also allowed efficient acoustic isolation against ambient noise at the QTF frequency. Finally, we performed an Allan deviation showing an absorption detection limit of $9 \times 10^{-9} \mathrm{~cm}^{-1}$ for $\mathrm{CO}_{2}$ detection around $1.5 \mu \mathrm{m}$.

Future work will include the optimization of the acoustic cavity and adaptation of the design for use with longwave infrared quantum cascade lasers.

Author Contributions: M.D. did the experiments; G.A. together with J.-M.M., R.L. and M.R. put in place the experimental setup and designed the quartz tuning fork and the recuperation cavity, as well as the microresonators; G.A. developed the analytical model; A.G. guided the work as PhD director.

Funding: M.D.'s PhD grant was funded by MirSense and Association Nationale de la Recherche Technologique. The project was partially funded by the FCS Campus Paris-Saclay under grant 2014-0455I (MICSI).

Conflicts of Interest: The authors declare no conflict of interest.

\section{References}

1. Bell, A.G. Upon the Production of Sound by Radiant Energy; Gibson Brothers, printers: Washington, DC, USA, 1881.

2. Kosterev, A.A.; Bakhirkin, Y.A.; Curl, R.F.; Tittel, F.K. Quartz-enhanced photoacoustic spectroscopy. Opt. Lett. 2002, 27, 1902-1904. [CrossRef] [PubMed]

3. Lee, M.; Jahng, J.; Kim, K.; Jhe, W. Quantitative atomic force measurement with a quartz tuning fork. Appl. Phys. Lett. 2007, 91, 023117. [CrossRef]

4. Patimisco, P.; Spagnolo, V.; Vitiello, M.; Scamarcio, G.; Bledt, C.; Harrington, J. Low-loss hollow waveguide fibers for mid-infrared quantum cascade laser sensing applications. Sensors 2013, 13, 1329-1340. [CrossRef] [PubMed]

5. Wysocki, G.; Kosterev, A.A.; Tittel, F.K. Influence of molecular relaxation dynamics on quartz-enhanced photoacoustic detection of $\mathrm{CO}_{2}$ at $\lambda=2 \mu \mathrm{m}$. Appl. Phys. B 2006, 85, 301-306. [CrossRef]

6. Dong, L.; Kosterev, A.A.; Thomazy, D.; Tittel, F.K. QEPAS spectrophones: Design, optimization, and performance. Appl. Phys. B 2010, 100, 627-635. [CrossRef]

7. Wu, H.; Dong, L.; Zheng, H.; Liu, X.; Yin, X.; Ma, W.; Zhang, L.; Yin, W.; Jia, S.; Tittel, F.K. Enhanced near-infrared QEPAS sensor for sub-ppm level H2S detection by means of a fiber amplified $1582 \mathrm{~nm}$ DFB laser. Sens. Actuators B Chem. 2015, 221, 666-672. [CrossRef]

8. Sampaolo, A.; Patimisco, P.; Giglio, M.; Vitiello, M.; Beere, H.; Richie, D.; Scamarcio, G.; Tittel, F.K.; Spagnolo, V. Improved tuning fork for terahertz Quartz-Enhanced Photoacoustic Spectroscopy. Sensors 2016, 16, 439. [CrossRef] [PubMed] 
9. Zeninari, V.; Parvitte, B.; Courtois, D.; Kapitanov, V.A.; Ponomarev, Y.N. Methane detection on the sub-ppm level with a near-infrared diode laser photoacoustic sensor. Infrared Phys. Technol. 2003, 44, 253-261. [CrossRef]

10. Tittel, F.K.; Sampaolo, A.; Patimisco, P.; Dong, L.; Geras, A.; Starecki, T.; Spagnolo, V. Analysis of overtone flexural modes operation in quartz-enhanced photoacoustic spectroscopy. Opt. Express 2016, 24, A682-A692. [CrossRef]

11. Patimisco, P.; Sampaolo, A.; Giglio, M.; dello Russo, S.; Mackowiak, V.; Rossmadl, H.; Cable, A.; Tittel, F.K.; Spagnolo, V. Tuning forks with optimized geometries for quartz-enhanced photoacoustic spectroscopy. Opt. Express 2019, 27, 1401-1415. [CrossRef] [PubMed]

12. Patimisco, P.; Sampaolo, A.; Dong, L.; Tittel, F.K.; Spagnolo, V. Recent advances in quartz enhanced photoacoustic sensing. App. Phys. Rev. 2018, 5, 011106. [CrossRef]

13. Wu, H.; Yin, X.; Dong, L.; Pei, K.; Sampaolo, A.; Patimisco, P.; Zheng, H.; Ma, W.; Zhang, L.; Yin, W.; et al. Simultaneous dual-gas QEPAS detection based on a fundamental and overtone combined vibration of quartz tuning fork. Appl. Phys. Lett. 2017, 110, 121104. [CrossRef]

14. Ma, Y.F.; Tong, Y.; He, Y.; Long, J.H.; Yu, X. Quartz-enhanced photoacoustic spectroscopy sensor with a small-gap quartz tuning fork. Sensors 2018, 18, 2047. [CrossRef] [PubMed]

15. Zheng, H.; Dong, L.; Yin, X.; Liu, X.; Wu, H.; Zhang, L.; Ma, W.; Yin, W.; Jia, S. Ppb-level QEPAS NO2 sensor by use of electrical modulation cancellation method with a high power blue LED. Sens. Actuators B Chem. 2015, 208, 173-179. [CrossRef]

16. Levy, R.; Duquesnoy, M.; Aoust, G.; Melkonian, J.M. New Signal Processing for Fast and Accurate QEPAS Measurements. In Proceedings of the 2018 IEEE International Ultrasonics Symposium (IUS), Kobe, Japan, 22-25 October 2018; pp. 1-3.

17. Liu, K.; Guo, X.; Yi, H.; Chen, W.; Zhang, W.; Gao, X. Off-beam quartz-enhanced photoacoustic spectroscopy. Opt. Lett. 2009, 34, 1594-1596. [CrossRef]

18. Rousseau, R.; Loghmari, Z.; Bahriz, M.; Chamassi, K.; Teissier, R.; Baranov, A.N.; Vicet, A. Off-beam QEPAS sensor using an 11- $\mu \mathrm{m}$ DFB-QCL with an optimized acoustic resonator. Optics Express 2019, 27, 7435-7446. [CrossRef] [PubMed]

19. Aoust, G.; Levy, R.; Verlhac, B.; Le Traon, O. Ultra high quality factor resonators operated in fluids. Sens. Actuators A Phys. 2018, 269, 569-573. [CrossRef]

20. Aoust, G. Développements de Sources Infrarouges et de Résonateurs en Quartz Pour la Spectroscopie Photoacoustique. Ph.D. Thesis, Université Paris-Saclay, Paris, France, 2016.

21. OOFELIE: Multiphysics by Open Engineering. Available online: https://www.open-engineering.com (accessed on 1 March 2019).

22. Aoust, G.; Levy, R.; Verlhac, B.; Le Traon, O. Optimal quality factor for tuning forks in a fluid medium. Sens. Actuators A Phys. 2016, 243, 134-138. [CrossRef]

23. Zheng, H.; Dong, L.; Sampaolo, A.; Wu, H.; Patimisco, P.; Yin, X.; Ma, W.; Zhang, L.; Yin, W.; Spagnolo, V.; et al. Single-tube on-beam quartz-enhanced photoacoustic spectroscopy. Opt. Lett. 2016, 41, 978-981. [CrossRef]

24. Wu, H.; Sampaolo, A.; Dong, L.; Patimisco, P.; Liu, X.; Zheng, H.; Yin, X.; Ma, W.; Zhang, L.; Yin, W.; et al. Quartz enhanced photoacoustic H2S gas sensor based on a fiber-amplifier source and a custom tuning fork with large prong spacing. Appl. Phys. Lett. 2015, 107, 111104. [CrossRef]

25. Aoust, G.; Levy, R.; Raybaut, M.; Godard, A.; Melkonian, J.M.; Lefebvre, M. Theoretical analysis of a resonant quartz-enhanced photoacoustic spectroscopy sensor. Appl. Phys. B 2017, 123, 63. [CrossRef]

26. Ma, Y.; Lewicki, R.; Razeghi, M.; Tittel, F.K. QEPAS based ppb-level detection of $\mathrm{CO}$ and $\mathrm{N}_{2} \mathrm{O}$ using a high power CW DFB-QCL. Opt. Express 2013, 21, 1008-1019. [CrossRef] [PubMed]

27. Dong, L.; Wright, J.; Peters, B.; Ferguson, B.A.; Tittel, F.K.; McWhorter, S. Compact QEPAS sensor for trace methane and ammonia detection in impure hydrogen. Appl. Phys. B 2012, 107, 459-467. [CrossRef]

(C) 2019 by the authors. Licensee MDPI, Basel, Switzerland. This article is an open access article distributed under the terms and conditions of the Creative Commons Attribution (CC BY) license (http:/ / creativecommons.org/licenses/by/4.0/). 